\title{
ANÁLISIS CUALITATIVO PARA LA MEJORA DEL SANTUARIO HISTÓRICO DE MACHU PICCHU: VISIÓN DE ACADÉMICOS VS. PROFESIONALES
}

\author{
QUALITATIVE ANALYSIS FOR THE IMPROVEMENT OF THE \\ HISTORIC SANCTUARY OF MACHU PICCHU: \\ VISION OF ACADEMICS VS PROFESSIONALS
}

\author{
Rafael Fuentes García \\ Universidad de Málaga, España \\ Angélica Arriola Miranda \\ Universidad San Martín de Porres, Perú
}

Aceptado: 3 de septiembre 2020

\section{RESUMEN}

El Santuario Histórico de Machu Picchu (SHM), elemento del patrimonio histórico material del Perú, declarado Patrimonio Mundial por la Unesco, es sin duda uno de los principales atractivos turísticos de Sudamérica; el cual, soporta una alta presión turística, problemas de saturación y riesgo de impacto en su conservación.

En el presente trabajo, se realiza una auditoría del nivel de calidad en la gestión turística del sitio utilizando el método Delphi. Se obtiene la visión de dos tipos de especialistas: académico y profesional en el sector turístico, sobre medidas para atenuar la situación descrita y así plantear lineamientos que tengan como fin mejorar la competitividad turística. Se requiere conocer los puntos de vista y propuestas de los investigadores universitarios y gestores de empresas turísticas con relación al futuro del SHM. Las conclusiones de la investigación, en función de la originalidad de sus objetivos y metodología, son de interés para la gestión de este patrimonio mundial.

Palabras clave: patrimonio turístico, Machu Picchu, Delphi, calidad turística, gestión turística sostenible, investigación cualitativa, eficiencia en la gestión turística.

\begin{abstract}
The Historic Sanctuary of Machu Picchu, an element of the tangible historical heritage of Peru, declared a World Heritage Site by Unesco, is undoubtedly one of the main tourist attractions in South America; which supports high tourist pressure, saturation problems and risk of impact on its conservation.
\end{abstract}

Este es un artículo Open Access bajo la licencia Creative Commons AtribuciónNoComercial-Compartirlgual 4.0

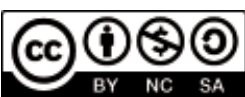


In the present work, an audit of the level of quality in the tourist management of the site is carried out using the Delphi method. The vision of two types of specialists is obtained: academic and professional, in the tourism sector, on measures to mitigate the situation described and thus propose guidelines that aim to improve tourism competitiveness. The points of view and proposals of university researchers and managers of tourism companies is relevant in relation to the future of the Historic Sanctuary of Machu Picchu. The conclusions of the research, depending on the originality of its objectives and methodology, are of interest for the management of this world heritage site.

Keywords: tourism heritage, Machu Picchu, Delphi, tourism quality, sustainable tourism management, qualitative research, tourism management efficiency.

El Santuario Histórico de Machu Picchu (SHM) está ubicado en la región Cusco, provincia de Urubamba, distrito de Machu Picchu, entre los ríos Cusichaca y Aobamba, en el punto de encuentro entre los Andes peruanos y la cuenca del Amazonas. Abarca 32592 hectáreas de laderas, picos y valles que rodean el monumento arqueológico de la ciudadela a más de 2400 m s. n. m. (Cátedra Unesco Patrimonio Cultural y Turismo Sostenible, USMP, 2020).

El SHM, es un complejo monumental constituido por una serie de testimonios arqueológicos inmersos en un espectacular paisaje montañoso de excepcional belleza, donde se destaca la ciudadela arqueológica de Machu Picchu, considerada uno de los mayores logros artísticos, arquitectónicos prehispánicos y el legado tangible más significativo de la civilización Inca. En efecto, la ciudadela de Machu Picchu es considerada la joya de la arquitectura incaica.

Los estudios sobre las ciudades incaicas señalan que se amoldaban a la disposición de la topografía del lugar.

Ciudades como Machu Picchu, levantada en lugares escabrosos, se adaptan a las sinuosidades del terreno y debe obedecer en su plano sólo a normas generales de planificación en sus sectores nucleares o centrales: las calles eran estrechas y graderías pétreas unían diversos niveles; no faltan las plazas y muchos recintos de claro destino sacro. (Kauffman, 1988, p. 565)

La ciudadela de Machu Picchu está erigida en planta en torno a tres grandes sectores: el sector civil, el sector religioso o sagrado y el sector agrícola, separados por la Plaza Central.

Existen diversas teorías acerca de la funcionalidad de Machu Picchu, desde aquellas que la proponen como lugar de origen, cuna o Pacaritambo (Tamputoco), hasta las que indican que fue lugar de refugio de los descendientes de los soberanos incas después de la conquista de los españoles y aún de las momias incas:

En Machu Picchu, hay un lugar que Hiram Bingham ha propuesto como «tumba real» y que pudo ser en efecto el lugar donde se cuidaba la momia de Pachacuti cuando ella no tenía que ir al Cusco para las festividades; ese lugar, según parece, pertenecía a ese Inca. (Com. pers. de J. H. Rowe, en base a documentos del Cusco, como se citó en Lumbreras, 2000, p. 54) 
Más allá de su portentosa arquitectura, Machu Picchu, y en general el despliegue de las obras de ingeniería incaica, representan la complejidad y el alcance del nivel de estrategia política, económica e ideológica de dominio del Imperio Inca sobre las diversas sociedades y territorios que conquistó (Cuadrao y Advíncula, 2017). Una verdadera joya patrimonial que es el mayor recurso turístico de todo Perú.

Además de su importancia como Patrimonio Cultural de la Nación y Patrimonio Mundial declarado por la UNESCO en la 7. a Sesión del Comité del Patrimonio Mundial, Florencia, deben considerarse sus valores ecológicos y medioambientales, ya que alberga una gran variedad de microclimas, hábitats y especies de flora y fauna (Cátedra Unesco Patrimonio Cultural y Turismo Sostenible, USMP, 2020). Se trata de un área reconocida internacionalmente por sus atributos naturales y culturales; presentando características geográficas excepcionales; formando parte de una estrecha faja de transición entre ecosistemas de montaña y de selva tropical húmeda, en las que se puede encontrar, desde cumbres nevadas por encima de los $6000 \mathrm{~m} \mathrm{~s}$. n. m., hasta la zona más húmeda y caliente del río Urubamba, por debajo de los 2000 m s. n. m. (Servicio Nacional de Áreas Naturales Protegidas [SERNANP], s.f.).

Dadas estas particularidades, diversas entidades del Estado peruano presentan jurisdicción sobre la gestión del SHM. Es un área natural protegida por el Gobierno del Perú, por intermedio de SERNANP (Decreto Supremo n. ${ }^{\circ}$ 001-81-AA del 08 de enero de 1981). A la riqueza natural excepcional, se le suma el valor histórico cultural, también reconocido y protegido por el Estado peruano mediante la Dirección Desconcentrada de Cultura de Cusco, organismo del Ministerio de Cultura, y a su vez del Área Funcional del Parque Arqueológico Nacional de Machu Picchu, ente que se encarga de la gestión y conservación del patrimonio cultural del Parque Arqueológico Nacional de Machupicchu-Santuario Histórico (SHM-PANM), garantizando su intangibilidad, inalienabilidad y autenticidad (Katahenggam, 2020), de acuerdo a estándares internacionales (Dirección Desconcentrada de Cultura Cusco, 2020). Asimismo, el SHM fue declarado el 9 de diciembre de 1983, sitio de Patrimonio Mundial Mixto -natural y cultural- por la UNESCO (SERNANP, s.f.). Esta superposición de instituciones marca un escenario complejo para la gobernanza interinstitucional y la efectividad de la gestión y protección de este bien cultural.

Desde el punto de vista turístico, como se ha señalado, es la principal y más importante atracción con la que cuenta el Perú y una de las más importantes de Latinoamérica; durante el año 2019 recibió 1585262 visitantes (Ministerio de Comercio Exterior y Turismo [MINCETUR], 2020).

Al ser el principal destino turístico del país, es obvio que el masivo arribo de visitantes, genera una serie de problemas en la preservación y conservación del santuario histórico, tanto en las manifestaciones culturales, monumentos y testimonios arqueológicos que lo constituyen como en el ecosistema y el medioambiente. La debilidad en la gestión turística también genera otro tipo de impactos como el incremento de precios que afecta también a residentes, o presión psicológica sobre la comunidad anfitriona, entre otros. Asimismo, ocasiona una serie de problemas en la prestación de los servicios turísticos, afectando marcadamente la calidad de la experiencia turística. Cabe mencionar que la Ley General de Turismo, establece que se debe regular la utilización con fines turísticos de los bienes inmuebles integrantes del Patrimonio Cultural de la Nación (MINCETUR, 2009), pero la gestión del número de visitantes ha colapsado, y los intentos por reorganizarla con un nuevo reglamento 
implementado a inicios del 2019 parece que están fracasando. Durante los últimos años, la falta de vigilancia y control dentro del santuario ha permitido una serie de transgresiones al reglamento, vulneraciones al patrimonio cultural, y hasta atentados serios contra el legado histórico del santuario (Lima Gris, 2019b).

Frecuentemente, en las investigaciones del sector turístico, se emplean técnicas cuantitativas para la realización de diagnósticos de destinos turísticos o propuestas de estrategias de mejora (Martínez Carazo, 2011). Esto, debido a la tendencia doctrinal, a la facilidad de su ejecución en series históricas sin trabajo fuera del que se tiene en el despacho, a la aparición de múltiples programas estadísticos para las ciencias sociales, a la valoración académica al utilizar métodos estadísticos complejos, etc. Pero, independientemente del motivo, las investigaciones basadas en estas técnicas cuantitativas suelen considerarse de mayor peso que las investigaciones basadas en técnicas cualitativas. No obstante, en esta investigación, se considera que el empleo de la técnica cualitativa o la técnica mixta como lo señala Gomez (2015) es lo más apropiado.

\section{Objetivos}

Se plantea, como primer objetivo, establecer la visión de especialistas del Perú en materia turística; sus consideraciones acerca de la situación y propuestas de mejora del SHM, recurso 'imán’ que atrae a turistas extranjeros al país. Es decir, conocer la percepción de 24 expertos sobre las acciones a tomar en el santuario histórico a mediano y largo plazo, con la finalidad de mejorar la competitividad del mismo, sobre la base de criterios de sostenibilidad social, económica y ambiental.

Un segundo objetivo, es determinar la importancia que los grupos de especialistas otorgan a las estrategias por implementar en el Santuario Histórico de Machu Picchu, teniendo en cuenta su ámbito de trabajo académico o profesional. Importancia referida a prácticas relacionadas con la gestión ambiental, servicios turísticos, infraestructuras, marketing, intervención pública o el producto turístico.

El tercer objetivo es analizar la importancia de acciones concretas (de un listado propuesto de 20), por implementar en el santuario histórico para lograr una mejora integral del recurso a medio y largo plazo.

El cuarto objetivo, es analizar los grados de coincidencia o divergencia de académicos y gestores, con el fin de establecer la visión específica de cada grupo.

\section{Revisión de la literatura científica}

El quehacer teórico acerca de la gestión de bienes culturales y patrimoniales, relacionado al turismo, tiene larga data. Es desarrollado por escuelas de pensamiento cada vez más sofisticadas y por un trabajo interdisciplinario ya consolidado.

En Europa se cuentan décadas de tradición, no solo en el debate científico en torno al patrimonio, sino también en la discusión y reflexión académica sobre sus diversas dimensiones en la formación de profesionales y en publicaciones especializadas (Calderón, 2002). El vicepresidente de la Fundación del Patrimonio Histórico 
de Castilla y León, se refiere a la «creciente formación de la sociedad en materia patrimonial, y del acercamiento de la misma a lo que ofrecen los testimonios de la cultura del pasado» (Hernández, 2002, p. 14).

En Latinoamérica, la reflexión en torno al patrimonio cultural viene madurando y tomando ribetes interesantes. En general, se observa una tendencia al cambio del marco institucional de gestión, inscrita en el doble movimiento interrelacionado de la reforma del Estado y del tránsito de lo nacional a lo local-municipal, y de allí, a lo empresarial-privado (Hayakawa, 2009).

En la década de los ochenta, el peso de la actuación sobre el patrimonio recaía en la figura del Estado, en cuanto a la concentración de funciones y roles asumidos en torno al bien cultural. Existía una posición confrontacional donde el patrimonio cultural debía ser defendido, protegido, salvado y las políticas proteccionistas marcaban un franco distanciamiento entre las comunidades y el Estado (Espinoza y Gildemeister, 2017). Todavía circulan publicaciones del Ministerio de Cultura intituladas Manual del Defensor del Patrimonio (Ministerio de Cultura, 2011). Esta interpretación del patrimonio llevaba a una marcada polarización de los agentes culturales (Marcone, 2019). Algunos casos son epónimos, como el descubrimiento en 1987 de las denominadas Tumbas Reales de Sipán, yacimiento arqueológico ubicado en el distrito de Saña, provincia de Chiclayo, región Lambayeque. El hallazgo, se pudo realizar en una acción de intervención de emergencia (entre efectivos de la policía y arqueólogos del Museo Nacional Brunning de Lambayeque) para detener la expoliación del mausoleo de este mandatario moche a manos de saqueadores de tumbas, denominados en jerga popular, huaqueros (Alva, 1996).

Los años 90 marcan el inicio de un nuevo estilo de acercamiento e intervención en el patrimonio; aparecen más y nuevas instituciones gestoras: se admite que el patrimonio debe ser administrado por un grupo amplio de sujetos multidisciplinares. Con el paso del tiempo, el estudio del patrimonio desde casillas estancas y con actores en franca polarización, se viene equilibrando, admitiendo una institucionalidad multidimensional en materia patrimonial, y arribando paulatinamente a un equilibrio entre el peso de la agencia sectorial y la voluntad territorial; entre la incidencia de lo global y la impronta de lo local. Así, hoy en día es reconocible el trinomio públicoprivado-comunitario (Hayakawa, 2009).

Actualmente, este trinomio muestra una inclinación hacia el lado comunitario, registrándose una tendencia al involucramiento de la comunidad en algunos proyectos de puesta en valor e investigación arqueológica (Cuadrao y Advíncula, 2017); aunque todavía no se pueda hablar de una política pública o de una posición oficial del Estado peruano. En estos contados -pero significativos casos- la agencia pública, presenta un acercamiento mucho más cuidadoso hacia el poblador local, equipada ya con marcos teóricos, métodos y técnicas para gestionar el patrimonio en un país lleno de conflictos sociales. Preparada para abordar la «problemática del patrimonio cultural enmarcada en el disenso» (Espinoza y Gildemeister, 2017).

Esta tendencia se plasma en la implementación del enfoque de puesta en uso social, que se registra y se ocupa en el Ministerio de Cultura ya desde el año 2005: «la perspectiva de la Puesta en Uso Social se empezó a trabajar por primera vez en el Perú en el ámbito Estatal, específicamente en el sector cultura, en el programa Qhapaq Ñan Sede Nacional en el año 2005» (Pino, 2019). 
La propuesta va presentando indicios audaces, se observa el viraje hacia una cogestión cultural entre Estado y comunidad (Cuadrao y Advíncula, 2017). Resulta interesante entender el patrimonio más como proceso, mediante el cual se acumula, renueva y producen percepciones alrededor de objetos, prácticas y conocimientos, administrados por una plataforma social que activa procesos de subjetivación, negociación política e identitaria. Por esta razón, el patrimonio se constituye en el espacio público idóneo para canalizar el conflicto y posibilitar la negociación; el ámbito idóneo para la formulación de valores y la obtención del consenso social.

Tratándose de un destino cuya oferta turística es principalmente de tipo cultural, resulta importante observar las particularidades del tejido social, institucional y político que enmarcan su gestión, que se complejiza aún más por la presión de la actividad turística que se cierne sobre muchos de los bienes culturales del país. Cabe recalcar que solo una mínima parte de los sitios patrimonio cultural del Perú son de interés turístico o presentan potencial turístico.

Sin embargo, la situación del Perú también ilustra una importante deficiencia de la relación entre turismo y cultura, a saber, la concentración en un número relativamente reducido de exponentes del patrimonio edificado, lo que provoca que otros emplazamientos y otras formas de cultura queden en cierto modo infrautilizados. De acuerdo con estudios llevados a cabo por el gobierno peruano, el 71\% de los turistas extranjeros viajan al país para visitar Machu Picchu; sin embargo, otros lugares de interés cultural siguen siendo auténticos desconocidos. (World Economic Forum, 2013)

La Carta de Pecs o Carta Europea de las Ciudades y Territorio de la Cultura y el Patrimonio, se refiere al patrimonio como «la suma de los cuidados que ponen los humanos sobre esos elementos irremplazables de su patrimonio común» (Lozano, 2002, p. 51). En el vasto universo de los bienes y elementos culturales presentes en Perú, se detecta un primer subconjunto que es el del Patrimonio Cultural de la Nación; es decir, aquellos bienes culturales que han sido declarados como tales por el Gobierno del Perú y han pasado a formar parte de su acervo patrimonial por medio de un catastro, registro o inventario llevado a cabo por el Estado en sus diversas instancias.

De acuerdo a la Ley n. 28296 (2004), bien integrante del Patrimonio Cultural de la Nación es:

Toda manifestación del quehacer humano -material o inmaterial- que por su importancia, valor y significado paleontológico, arqueológico, arquitectónico, histórico, artístico, militar, social, antropológico, tradicional, religioso, etnológico, científico, tecnológico o intelectual, sea expresamente declarado como tal o sobre el que exista la presunción legal de serlo. Dichos bienes tienen la condición de propiedad pública o privada con las limitaciones que establece la presente Ley.

Del conjunto de bienes que integran el Patrimonio Cultural de la Nación, destacan los 12 elementos integrados a la Lista del Patrimonio Mundial de la Unesco, ocho culturales, dos naturales y dos mixtos (Cátedra Unesco Patrimonio Cultural y Turismo Sostenible, USMP, 2020). Entre ellos, el SHM, sobre el cual se despliega una enorme presión turística

La relación entre turismo internacional y cultura es especialmente estrecha en el Perú. Tal y como apunta el Informe de competitividad de viajes y turismo de Perú, el país alberga algunas de las 
atracciones naturales y culturales más importantes del mundo, en particular, los yacimientos arqueológicos de Machu Picchu y Cusco, mundialmente conocidos. (Organización Mundial de Turismo [OMT], 2016)

\section{Sobre la gestión turística sostenible en el SHM}

El concepto de «turismo sostenible», acuñado en la Cumbre de Río Eco92, engloba el turismo de naturaleza, ecoturismo, turismo rural, turismo alternativo, agroturismo y cualquier otra actividad turística que cumpla con las bases de sostenibilidad sociocultural, económica y ambiental.

La OMT (2004) se refiere a desarrollo o gestión sostenible del turismo, que implica establecer un balance entre las dimensiones ambientales, económicas y socioculturales del desarrollo turístico, necesarios para garantizar su sostenibilidad a largo plazo. En ese sentido, el turismo sostenible debe cumplir con las siguientes características:

- Dar uso óptimo a los recursos ambientales por ser elementos fundamentales en el desarrollo turístico, manteniendo los procesos ecológicos esenciales y ayudando a conservar los recursos naturales y la diversidad biológica.

- $\quad$ Respetar la autenticidad sociocultural de las comunidades anfitrionas, conservar sus activos culturales arquitectónicos y vivos, con sus valores tradicionales; y contribuir al entendimiento y a la tolerancia intercultural.

- Asegurar actividades económicas viables a largo plazo, que reporten a todos los agentes beneficios socioeconómicos bien distribuidos, con oportunidades de empleo estable, e ingresos y servicios sociales para las comunidades anfitrionas y así contribuir a reducir la pobreza.

Es necesario elevar los estándares de calidad en la gestión del SHM, para contrarrestar los problemas como deterioro del patrimonio natural, cultural y decaimiento de la experiencia turística.

La gestión turística debe abordar un conjunto de dimensiones que garanticen una mejor y más provechosa practica turística del elemento Machu Picchu, garantizando la conservación y preservación del patrimonio cultural, así como una experiencia turística satisfactoria para el visitante y, sobre todo, con la integración de la comunidad (Ypeij et al., 2020, pp. 442-444). Una adecuada gestión turística necesita referentes o puntos de medición para realizar el monitoreo y vigilancia de los procesos implementados; detectar si los resultados, que se van generando, son los esperados.

La planificación y gestión turística de territorios requiere cada vez más de instrumentos que permitan una visión más global y esencialmente comparada entre destinos, para identificar tendencias y promover lógicas de desarrollo equilibradas. Por este motivo proliferan las propuestas de indicadores complejos que proporcionan una comprensión más global e integrada del fenómeno turístico. (López et al., 2018, p. 435) 
En la presente investigación, los indicadores e índices marcan estándares que permiten observar y evaluar la situación de manera rápida, ofreciendo un solo valor comparable entre diferentes casos. La auditoría de calidad en la gestión turística se realizó en las siguientes dimensiones: gestión ambiental, gestión de servicios turísticos, infraestructura pendiente, marketing, intervención pública y producto turístico.

La gestión ambiental es definida por el Consejo Nacional del Ambiente como un proceso permanente y continuo orientado a administrar los intereses y recursos relacionados con los objetivos de la Política Nacional Ambiental, con el fin de alcanzar una mejor calidad de vida para la población, el desarrollo de las actividades económicas, el mejoramiento del ambiente urbano y rural, así como la conservación del patrimonio natural del país, entre otros objetivos (Consejo Nacional del Ambiente [CONAM], 2005). En este campo, se pidió opinión a los expertos participantes sobre las siguientes líneas de intervención: posibilidades de mejora de la gestión sostenible del recurso; necesidad de rediseñar la política de gestión de flujos turísticos dentro del espacio patrimonial; y, necesidad de implementar una política de conservación preventiva.

Sobre gestión de servicios turísticos, en el caso peruano, la Ley General de Turismo n. ${ }^{\circ}$ 29408, define como prestadores de servicios turísticos a «las personas naturales o jurídicas que participan en la actividad con el objeto principal de proporcionar servicios turísticos directos de utilidad básica e indispensable para el desarrollo de las actividades de los turistas» (MINCETUR, 2009). En los anexos de la misma ley se establecen los tipos de servicios turísticos que pueden ser prestados por el empresariado privado mediante negocios turísticos, reglamentados en cada caso: hospedaje; agencias de viaje y turismo; agencias operadoras de viajes y turismo; transporte turístico; guías de turismo; organización de congresos, convenciones y eventos; orientadores turísticos; restaurantes; centros de turismo termal y/o similares; turismo de aventura, ecoturismo o similares; y servicios de juegos de casino y máquinas tragamonedas.

En el ámbito de la generación, prestación y gestión de servicios turísticos, se consultó a los expertos sobre las siguientes líneas de acción a implementar en el santuario: importancia de mejorar la prestación de información turística, urgencia de mejorar los servicios (baños) públicos y necesidad de garantizar atención médica de primeros auxilios a los visitantes.

Sobre la infraestructura pendiente, la Ley General de Turismo del Perú, en su artículo 20, estipula:

El Ministerio de Comercio Exterior y Turismo (Mincetur) promueve la ejecución de proyectos de inversión turística a nivel nacional que coadyuven al desarrollo económico y social del país y a la preservación del patrimonio cultural y natural, fortaleciendo la consolidación de productos turísticos sostenibles e impulsando esquemas de financiamiento mixtos que consideren inversión pública y privada. (MINCETUR, 2009)

Los servicios turísticos públicos son aquellos garantizados por el Estado para procurar una mejor experiencia turística. Generalmente, son proveídos mediante proyectos de inversión pública en turismo, actualmente formulados y ejecutados bajo el Sistema Nacional de Programación Multianual de Inversiones Invierte.Pe (Ministerio de Economía y Finanzas [MEF], s.f.). Son considerados servicios turísticos públicos en el Perú: servicios de 
observación; de orientación turística; de información turística; de interpretación cultural; de disfrute del paisaje; de descansos (para recorridos largos); de embarque y desembarque para uso turístico; de acceso directo al recurso/atractivo, para el recorrido interno del recurso/atractivo; para exposición de muestras culturales; para exposición de flora y fauna silvestre; para estacionamiento de transporte turístico (MEF, 2011).

En cuanto al análisis de la estructura e infraestructura turística, se recogió la opinión de los expertos acerca de las siguientes líneas de acción: necesidad de crear nuevas carreteras de acceso a Machu Picchu; desarrollo de infraestructuras y programas de actividades culturales; dotación de un buen centro de interpretación turística en el santuario histórico.

Con relación al marketing de destinos turísticos, la Ley General de Turismo (MINCETUR, 2009) establece que las actividades de promoción turística de los sitios deben ser llevadas a cabo de acuerdo con las políticas sectoriales en turismo, y alineadas con estrategias y planes de promoción turística a nivel país. En cuanto a las actividades de marketing turístico, se exploraron las siguientes propuestas: realizar campañas de promoción para captar visitas en temporada baja; mejorar la información que se brinda al visitante, así como fomentar el uso de aplicaciones de las TIC; prioridad en desarrollar una estrategia de marketing para una comercialización del bien más sostenible.

Dada la complejidad de la trama administrativa que se yergue sobre la jurisdicción del monumento y considerando además que el desarrollo de la actividad turística constituye una función compartida por los distintos niveles de gobierno (MEF, 2011), se presentan retos y desafíos para la cogobernanza y la gestión interinstitucional. En esta dimensión se consultó la opinión de los expertos con relación a las siguientes líneas de intervención: la necesidad de mejorar las relaciones con la población local, así como mejorar la identificación social y cultural con el recurso; la urgencia de potenciar la empleabilidad entre la población local; la importancia de concienciar a la población local para que se sienta identificada con el destino turístico y la necesidad de mejorar la gobernanza entre los distintos entes interinstitucionales que participan en la gestión

Por último, se analizó el producto turístico, que se define como el conjunto de componentes, tangibles e intangibles, que incluyen recursos, atractivos, infraestructura, actividades recreativas, imágenes y valores simbólicos, para satisfacer motivaciones y expectativas, siendo percibidos como una experiencia turística (MEF, 2011). En este eje se exploraron las siguientes líneas de intervención: eventual incremento de los precios de las entradas para evitar la masificación; creación de productos complementarios y diversificación de la visita en el mismo espacio turístico-patrimonial; mejora de la interpretación del patrimonio y priorización de acciones que generen un mayor impacto económico en la zona.

\section{Metodología}

El método Delphi tiene su origen en los estudios realizados por la Rand Corporation en Estados Unidos a finales de la década de los 40, y fue desarrollado con el propósito de obtener una medida fiable del consenso entre un grupo de expertos, con la ventaja de evitar la confrontación directa entre ellos. Se trata de un método y técnica de investigación aplicado para obtener una opinión consensuada de un grupo de personas, reconocidas 
como «expertos, panelistas o informantes», aunque aplicaciones posteriores restaron importancia a la obtención del consenso, siendo el objetivo primordial la obtención de información cualitativa fiable de parte de los expertos (Martinez del Vas, 2017).

El método Delphi se utiliza en el quehacer turístico desde hace al menos cuarenta años, principalmente para la gestión de eventos, el pronóstico de la evolución de la demanda turística y las tendencias del mercado (Pulido y Ruiz, 2017). Sus aplicaciones en el campo turístico son numerosas, principalmente orientadas a prospectar escenarios futuros y tendencias. Recientemente, los estudios Delphi estuvieron centrados en la identificación de factores influyentes o definitorios en diferentes áreas del turismo (Martinez del Vas, 2017).

Se aplicó el método Delphi en dos rondas asincrónicas de preguntas formuladas a 24 expertos. En la primera vuelta respondieron 24 y en la segunda vuelta 22 (dos de ellos no siguieron con el estudio propuesto); resultado más que positivo para procesos metodológicos de este tipo.

Un aspecto clave en esta metodología es la selección de los expertos (Pulido y Ruiz, 2017). En este caso, el panel se integró con participantes seleccionados en función de sus conocimientos y su alta cualificación en el sector turístico, tanto en el sector público (cargos de la administración pública, técnicos públicos, etc.) como privado (empresariado privado reconocido, representantes del sector hostelero, etc.). El 45\% son académicos, miembros de grupos de investigación en el Perú; 9\% son empresarios del sector turístico y 18\% son gestores públicos, con representatividad en el ICOMOS, Ministerio de Cultura, organismos de ecoturismo y turismo de naturaleza, entre otros. El grado de conocimiento de los expertos participantes es muy alto, dado que el $72 \%$ trabaja más de 20 años en el sector turístico. El 100\% de los participantes conoce el SHM y su problemática.

La primera vuelta, que se inició en noviembre de 2019, integró un cuestionario dividido en seis apartados: 1. gestión ambiental, 2. servicios turísticos, 3. infraestructuras, 4. marketing, 5. intervención pública y 6. producto turístico (cada apartado se dividió en tres o cuatro preguntas específicas). La segunda vuelta se realizó en abril de 2020, replicó a la primera. Para garantizar los resultados durante la segunda vuelta, se integró un cuestionario individual para cada participante en el que se mostraba el resultado conjunto de los expertos en esa primera vuelta y la posición particular de cada especialista según su respuesta.

Se diseñó una base de datos en SPSS v.20 y se volcó la información que se trabajó en tablas dinámicas de Excel.

Posteriormente se contactó a los expertos vía teléfono y correo electrónico para explicarles el objetivo del trabajo y el método a utilizar; asimismo, se les brindó la siguiente información: presentación de las personas a cargo de la investigación y filiación institucional respectiva; objetivos de la investigación; naturaleza y características del método Delphi; número de veces que se debe responder al cuestionario; procesamiento de la información recibida; aportes y beneficios de su participación. En la Tabla 1 se muestra la ficha técnica de la investigación. 
Tabla 1

Ficha técnica del método Delphi

\begin{tabular}{ll}
\hline Ámbito de estudio & Santuario Histórico de Machu Picchu \\
Tamaño de la muestra & 24 cuestionarios en la primera vuelta \\
& 22 cuestionarios en la segunda vuelta \\
Fecha del trabajo empírico & Inicio: diciembre 2019 \\
& Fin: abril de 2020 \\
Tipo de estudio & Método Delphi \\
\hline
\end{tabular}

Nota. Elaboración propia.

\section{Resultados generales de la investigación}

Luego del análisis cualitativo de las respuestas de los participantes (24 expertos consultados en la primera vuelta), se procedió al envío, a cada una de las personas que participaron en el estudio, de los resultados globales obtenidos por el conjunto de los expertos y de la valoración individual a cada una de las estrategias e ítems propuestos. Cabe reiterar que de los 24 expertos que contestaron en la primera vuelta, lo hicieron 22 en la segunda vuelta, resultado más que positivo para procesos metodológicos de este tipo. Los resultados de la segunda vuelta se tabularon y analizaron, tal y como se realizó en la primera fase del presente trabajo, según los siguientes criterios:

- Por máxima puntuación, utilizando el promedio de las respuestas en esta segunda vuelta, sobre todas las respuestas obtenidas a cada una de las premisas planteadas.

- Por máximo consenso, utilizando la desviación estándar de las respuestas en esta segunda vuelta, sobre todas las respuestas obtenidas a cada una de las premisas planteadas.

\section{Por máxima puntuación}

A continuación y de forma enunciativa, se presentan los resultados obtenidos según las valoraciones definitivas de los expertos consultados, distinguiéndolos en bloques dependiendo de la puntuación otorgada, según la siguiente distribución:

\section{Máxima puntuación. Valoración de más de 7.4 puntos sobre 9.}

- Mejorar la gobernanza de los entes que participan en la gestión.

- Mejorar la información al visitante y el uso de las TIC.

- Rediseñar la gestión de flujos turísticos dentro del espacio patrimonial. 
Puntuación alta. Valoración de 6.5 a 7.4 puntos.

- Diseñar una política de conservación preventiva.

- Mejorar las relaciones con la población local.

- Potenciar la empleabilidad entre la población local.

- Apuesta por acciones que generen un mayor impacto económico.

- Concienciar a la población local para identificación con el destino turístico.

- Mejorar la gestión sostenible del recurso.

- Dotar de atención médica de primeros auxilios.

\section{Puntuación media. Valoración de 5 a 6.5 puntos.}

- Dotar de un buen centro deinterpretación turística.

- Mejorar la interpretación del patrimonio.

- Desarrollar infraestructuras y programas culturales.

- Mejorar la información turística.

- Crear productos complementarios y diversificar la visita.

- Estrategia de marketing para una comercialización del bien más sostenible.

- Mejorar los servicios (baños) públicos.

\section{Mínima puntuación. Valoración de 4 a 5 puntos.}

- Crear nuevas carreteras de acceso a Machu Picchu.

- Subir los precios de las entradas para evitar la masificación.

- Campañas de promoción para captar visitas en temporada baja. 
Tabla 2

Valoración media otorgada por expertos

\section{VALORACIÓN MEDIA GLOBAL}

Mejorar la gobernanza de los entes que participan en la gestión.

Mejorar la información al visitante y el uso de las TIC.

Rediseñar la gestión de flujos turísticos dentro del espacio.

7.45

Diseñar una política de conservación preventiva.

Mejorar las relaciones con la población local.

Potenciar la empleabilidad entre la población local.

Apuesta por acciones que generen un mayor impacto económico.

Concienciar a la población local para identificación con el destino turístico. 6.75

Mejorar la gestión sostenible del recurso.

6.60

Dotar de atención médica de primeros auxilios.

6.60

Dotar de un buen centro de interpretación turística. $\quad 6.30$

$\begin{array}{ll}\text { Mejorar la interpretación del patrimonio. } & 6.20\end{array}$

Desarrollar infraestructuras y programas culturales. $\quad 6.10$

$\begin{array}{ll}\text { Mejorar la información turística. } & 6.00\end{array}$

Crear productos complementarios y diversificar la visita. $\quad 6.05$

Estrategia de marketing para una comercialización del bien más sostenible. $\quad 5.95$

Mejorar los servicios (baños) públicos.

Crear nuevas carreteras de acceso a Machu Picchu. $\quad 4.80$

Subir los precios de las entradas para evitar la masificación. $\quad 4.30$

Campañas de promoción para captar visitas en temporada baja. $\quad 4.20$

Nota. Elaboración propia

\section{Por máximo consenso}

Tal y como se desarrolló en el apartado anterior, a continuación y de forma enunciativa, se presentan los resultados obtenidos según las valoraciones de los expertos consultados en la segunda vuelta, distinguiéndolos en bloques dependiendo del grado de acuerdo en sus respuestas a las reflexiones señaladas, según la siguiente distribución:

\section{Máximo acuerdo. Desviación estándar inferior a 1.}

- Dotar de atención médica de primeros auxilios.

- Diseñar una política de conservación preventiva.

- Rediseñar la gestión de flujos turísticos dentro del espacio patrimonial.

- Mejorar la información al visitante y el uso de las TIC.

- Mejorar la gobernanza de los entes que participan en la gestión. 
Acuerdo alto. Desviación estándar de 1 a 1.30.

- Crear productos complementarios y diversificar la visita.

- Mejorar las relaciones con la población local.

- Potenciar la empleabilidad entre la población local.

- Mejorar la gestión sostenible del recurso.

- Concienciar a la población local para identificación con el destino turístico.

- Estrategia de marketing para una comercialización del bien más sostenible.

\section{Acuerdo bajo. Desviación estándar de 1.30 a 1.50.}

- Dotar de un buen centro de interpretación turística.

- Desarrollar infraestructuras y programas culturales.

- Mejorar la información turística.

- Apuesta por acciones que generen un mayor impacto económico.

- Mejorar la interpretación del patrimonio.

- Mejorar los servicios (baños) públicos.

\section{Desacuerdo. Desviación estándar de más de 1.50.}

- Campañas de promoción para captar visitas en temporada baja.

- Subir los precios de las entradas para evitar la masificación.

- Crear nuevas carreteras de acceso a Machu Picchu. 
Tabla 3

Grado de consenso de respuestas otorgadas por los expertos

\section{DESVIACIÓN DE LAS RESPUESTAS}

Dotar de atención médica de primeros auxilios.

0.82

Diseñar una política de conservación preventiva.

0.83

Rediseñar la gestión de flujos turísticos dentro del espacio patrimonial.

0.89

Mejorar la información al visitante y el uso de las TIC.

0.99

Mejorar la gobernanza de los entes que participan en la gestión. $\quad 0.88$

Crear productos complementarios y diversificar la visita. $\quad 1.05$

Mejorar las relaciones con la población local. $\quad 1.15$

Potenciar la empleabilidad entre la población local. $\quad 1.18$

Mejorar la gestión sostenible del recurso. $\quad 1.19$

Concienciar a la población local para identificación con el destino turístico. $\quad 1.25$

Estrategia de marketing para una comercialización del bien más sostenible. $\quad 1.28$

Dotar de un buen centro de interpretación turística. $\quad 1.30$

Desarrollar infraestructuras y programas culturales. $\quad 1.33$

Mejorar la información turística. $\quad 1.34$

Apuesta por acciones que generen un mayor impacto económico.

Mejorar la interpretación del patrimonio. $\quad 1.36$

Mejorar los servicios (baños) públicos. $\quad 1.37$

Campañas de promoción para captar visitas en temporada baja. $\quad 1.79$

Subir los precios de las entradas para evitar la masificación. $\quad 2.15$

Crear nuevas carreteras de acceso a Machu Picchu. $\quad 2.57$

Nota. Elaboración propia.

\section{Media y desviación estándar}

En la Tabla 4 se muestra el análisis comparativo de la valoración otorgada por el conjunto de los expertos a las reflexiones planteadas en la segunda vuelta; así como, el grado de consenso o divergencia en sus respuestas.

De acuerdo a los resultados, producto del análisis, se llega a las siguientes conclusiones:

- Son medidas prioritarias para mejorar el recurso turístico SHM, según respuestas de los expertos consultados:

1. Mejorar la gobernanza de los entes públicos que participan en la gestión del santuario; con una valoración media de 8.15 puntos y una desviación estándar de 0.88 , lo que significa alta valoración de la necesidad de su puesta en marcha y coincidencia entre el conjunto de los expertos consultados.

2. Es necesario mejorar la información que se pone a disposición del visitante por medio de las TIC; con una valoración media de 7.65 puntos y una desviación estándar de 0.99 .

3. Es importante, rediseñar la gestión de los flujos turísticos dentro del espacio patrimonial; con una valoración media de 7.45 puntos y una desviación estándar de 0.89 . 
Tabla 4

Valoración y grado de consenso en la segunda vuelta

\begin{tabular}{|c|c|c|}
\hline & Valoración & Desv. estándar \\
\hline Mejorar la gobernanza de los entes que participan en la gestión. & 8.15 & 0.88 \\
\hline Mejorar la información al visitante y el uso de las TIC. & 7.65 & 0.99 \\
\hline Rediseñar la gestión de flujos turísticos dentro del espacio. & 7.45 & 0.89 \\
\hline Diseñar una política de conservación preventiva. & 7.20 & 0.83 \\
\hline Mejorar las relaciones con la población local. & 7.20 & 1.15 \\
\hline Potenciar la empleabilidad entre la población local. & 7.15 & 1.18 \\
\hline Apuesta por acciones que generen un mayor impacto económico. & 6.85 & 1.35 \\
\hline Concienciar a la población local para identificación con el destino turístico. & 6.75 & 1.25 \\
\hline Mejorar la gestión sostenible del recurso. & 6.60 & 1.19 \\
\hline Dotar de atención médica de primeros auxilios. & 6.60 & 0.82 \\
\hline Dotar de un buen centro de interpretación turística. & 6.30 & 1.30 \\
\hline Mejorar la interpretación del patrimonio. & 6.20 & 1.36 \\
\hline Desarrollar infraestructuras y programas culturales. & 6.10 & 1.33 \\
\hline Mejorar la información turística. & 6.00 & 1.34 \\
\hline Crear productos complementarios y diversificar la visita. & 6.05 & 1.05 \\
\hline Estrategia de marketing para una comercialización del bien más sostenible. & 5.95 & 1.28 \\
\hline Mejorar los servicios (baños) públicos. & 5.75 & 1.37 \\
\hline Crear nuevas carreteras de acceso a Machu Picchu. & 4.80 & 2.57 \\
\hline Subir los precios de las entradas para evitar la masificación. & 4.30 & 2.15 \\
\hline Campañas de promoción para captar visitas en temporada baja. & 4.20 & 1.79 \\
\hline
\end{tabular}

Nota Elaboración propia.

4. Es significativa la necesidad de mejorar el diseño de una política de conservación ambiental preventiva; con una valoración media de 7.20 puntos y una desviación estándar de 0.83.

5. Es necesario incidir en mejorar la relación con la población local y los efectos provocados por los visitantes al recurso; con una valoración media de 7.20 puntos y una desviación estándar de 1.15.

Se obtiene una alta valoración en la necesidad de poner en marcha estas medidas, y una notable coincidencia en el conjunto de los expertos consultados.

- Las medidas de segundo grado en ejecución, pero con muy alta importancia, según los expertos consultados, son las siguientes:

1. Es necesario potenciar las acciones de mejora de creación de empleo para la población local; con una valoración media de 7.15 puntos y una desviación estándar de 1.18.

2. Se considera importante mejorar aspectos relacionados con la apuesta por acciones que generen un mayor impacto económico en el entorno social del recurso. 
3. Se indica como relevante concienciar a la población local de Cusco y Aguas Calientes en la importancia del recurso para su vida; con una valoración media de 6.75 puntos y una desviación estándar de 1.25.

4. Mejorar la gestión sostenible del recurso, del destino turístico, es un elemento considerado como esencial. Con una valoración media de 6.60 puntos y una desviación estándar de 1.19.

5. Necesaria la implementación de la atención médica de primeros auxilios en el mismo SHM; con una valoración media de 6.60 puntos y una desviación estándar de 0.82 .

Se comprueba cómo los expertos han otorgado una alta valoración a la necesidad de poner en marcha las cinco propuestas anteriores, además de una aceptable coincidencia en la opinión del conjunto de los expertos consultados.

- Elementos considerados importantes, pero no estratégicos en la valoración otorgada por los expertos consultados, son los siguientes: dotar de un buen centro de interpretación turística (6.30); mejorar la interpretación del patrimonio (6.20); desarrollar infraestructuras y programas culturales (6.10); crear productos complementarios y diversificar la visita (6.05); mejorar la información turística (6.00). Además, todas ellas, excepto la propuesta de crear productos complementarios y diversificar la visita, tienen una desviación estándar mayor a 1.30, por lo que no existe acuerdo significativo entre los expertos consultados.

- Son cinco aspectos con menor valoración: apuesta por una estrategia de marketing para la comercialización del bien más sostenible; la mejora de los servicios (baños) públicos; la creación de nuevas carreteras de acceso a Machu Picchu; la subida de los precios de las entradas para evitar la masificación; y la realización de campañas de promoción para captar visitas en temporada baja, siendo estos tres últimos los menos valorados, con una puntuación por debajo de cinco. Además, se comprueba que no existe una opinión homogénea entre los entrevistados, ya que cuatro de las anteriores propuestas tienen una desviación estándar por encima de 1.3 puntos, observándose divergencia en las opiniones sobre la necesidad de crear una carretera de acceso Aguas Calientes-Machu Picchu, pero con una baja valoración media.

\section{Comparación primera y segunda vuelta}

La comparación de los resultados de la valoración otorgada en primera y segunda vuelta, permiten destacar cambios significativos muy puntuales, por lo que la segunda vuelta solo ha perfilado ciertas tendencias.

A nivel global, la valoración media en los veinte ítems estudiados, pasa de 5.99 puntos en la primera vuelta a 6.36 puntos en la segunda vuelta. Se observa un incremento de 0.38 puntos más en la valoración definitiva otorgada al conjunto de las propuestas señaladas. 


\section{Figura 1}

Comparación de resultados de la valoración otorgada en primera y segunda vuelta

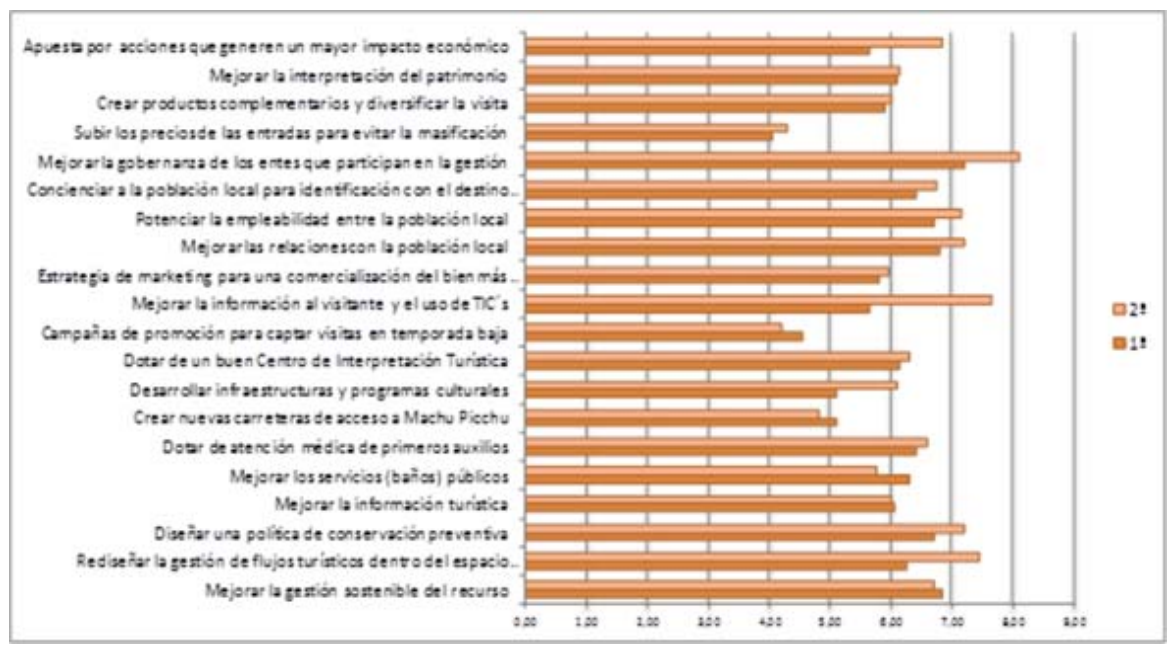

Nota. Elaboración propia.

Los cambios más significativos se producen de la siguiente manera:

- Incremento significativo en la valoración de los expertos consultados, con más de un punto en la segunda vuelta, en lo siguiente: mejora de la información al visitante y uso de las TIC (es la mayor variación observada, con más 2 puntos); rediseñar la gestión de flujos turísticos dentro del espacio patrimonial (más de 1.2 puntos); apuesta por acciones que generen un mayor impacto económico (más de 1.2 puntos); desarrollo de infraestructuras y programas culturales en el entorno del recurso (más de 1 punto).

- Las propuestas con mayor cambio de la valoración, efectuada por los especialistas consultados en la reflexión final, son las siguientes: mejora de la gobernanza de los entes que participan en la gestión; diseño de una política de conservación preventiva; potenciar la empleabilidad entre la población local; mejora de las relaciones con la población local y concienciación a la población local para identificación con el destino turístico. En estas propuestas se incrementa la valoración; así tenemos: 0.95 puntos en mejora de la gobernanza y 0.35 puntos en desarrollo de un plan de concienciación local.

- Las propuestas en las que hay mínima variación en la valoración de los expertos consultados son las siguientes: subida de los precios de las entradas para evitar la masificación; dotar de atención médica de primeros auxilios; estrategia de marketing para una comercialización del bien más sostenible; dotar de un buen centro de interpretación turística; creación de productos complementarios y diversificación de la visita; mejora de la interpretación del patrimonio. Con una subida media de solo 0.17 puntos en estas propuestas.

- En cinco propuestas se ve disminuida la valoración de los expertos consultados en la reflexión final con respecto a la inicial: mejora de la información turística; mejora de la gestión sostenible del recurso; creación de nuevas carreteras de acceso a Machu Picchu; realización de campañas de promoción para captar visitas en temporada baja; mejora de los servicios (baños) públicos en el recinto histórico. En esta última propuesta, 
los expertos disminuyen su valoración en la segunda vuelta, pasando de 6.30 a 5.75 puntos, en la reflexión final.

Se comprueba que no se produce una variación en el primer puesto del ranking de valoración media, la mejora de la gobernanza, pero sí en el último, que pasa de ser la subida de los precios de las entradas para evitar la masificación en la primera reflexión, a la propuesta de diseñar campañas de promoción para captar visitas en temporada baja, en la segunda.

En las ocho principales propuestas realizadas por los expertos para la mejora del recurso, en todas se ha comprobado cómo la recomendación de su puesta en funcionamiento se incrementa en la reflexión final, concretamente, las relacionadas con la mejora de la gobernanza de los entes que participan en la gestión (más de 0.95); mejora de la información al visitante y el uso de las TIC (con un sorprendente incremento de dos puntos sobre la valoración inicial); rediseñar la gestión de flujos turísticos dentro del espacio patrimonial (más de 1.20); necesidad de diseñar una política de conservación preventiva (más de 0.5); realización de actuaciones de mejora de las relaciones con la población local (más de 0.4); potenciación de la empleabilidad entre la población local (más de 0.45 ); apuesta por acciones que generen un mayor impacto económico (más de 1.2); realización de campañas de concienciación a la comunidad anfitriona para identificación con el destino turístico (más de $0.35)$.

En el análisis de la desviación en las opiniones de los especialistas consultados, se observan algunos cambios significativos; aumentan o disminuyen sus medias, con una disminución media de 0.3 puntos en la desviación estándar de la primera vuelta con respecto a la segunda vuelta.

\section{Figura 2}

Comparación de la coincidencia en la opinión de la primera y segunda vuelta

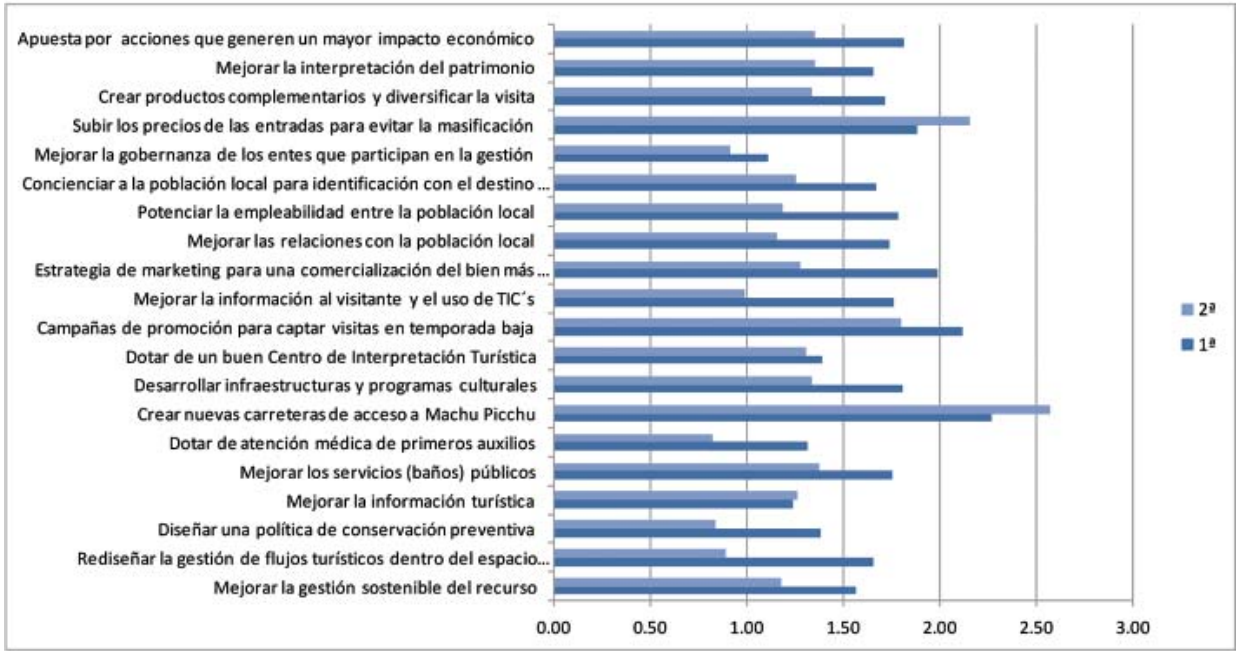

Nota. Elaboración propia. 
Se produce un mayor acercamiento o consenso entre todos los expertos, de este modo, si se compara la desviación estándar de la primera vuelta con la segunda, en diecisiete de las veinte reflexiones planteadas se produce una disminución. Se destaca lo siguiente:

- Las mayor variación en las opiniones de los expertos se producen en las siguientes propuestas: mejora de la información al visitante y el uso de las TIC; rediseñar la gestión de flujos turísticos dentro del espacio patrimonial; estrategia de marketing para una comercialización del bien más sostenible; crear productos complementarios y diversificar la visita; potenciar la empleabilidad entre la población local. Todas con una disminución de la desviación estándar muy significativa.

- El segundo bloque de reflexiones, con tendencia a un mayor acercamiento en las opiniones de los expertos comprende: mejorar las relaciones con la población local; diseñar una política de conservación preventiva; dotar de atención médica de primeros auxilios; desarrollar infraestructuras y programas culturales; apuesta por acciones que generen un mayor impacto económico; concienciar a la población local para identificación con el destino turístico.

\section{Comparación entre académicos y profesionales}

Uno de los objetivos de la presente investigación es conocer si difieren en percepción, los académicos y profesionales turísticos de Lima, sobre las mejoras a realizar en el SHM. Por lo cual, y como se indicó en la metodología, se seleccionaron a personas especialistas en esta temática en los dos ámbitos sectoriales señalados.

Se presentaron, hasta el momento, las conclusiones de las opiniones de los 24 expertos en la primera vuelta y de los 22 expertos en la segunda vuelta, sin segmentar de acuerdo al ámbito turístico de dichos participantes. Por lo tanto, es necesario analizar la casuística particular de la visión de los expertos académicos y profesionales en la gestión turística, pormenorizado cada uno de los aspectos, así como la valoración y el consenso.

Del análisis comparativo, tanto en su valoración como en el consenso entre académicos y profesionales en el sector turístico, se establece que los académicos otorgan mayores valoraciones que los profesionales, ya que la valoración media de los primeros es de 6.9 y 6.0 de los segundos.

Como conclusiones generales se observan las siguientes:

- Tanto académicos como profesionales coinciden en que lo más importante es la mejora de la gobernanza de los entes públicos que participan en la gestión del recurso.

- Las siguientes propuestas muestran mayor coincidencia en la necesidad de ejecución: mejora de la información al visitante y el uso de las TIC; necesidad de rediseñar la gestión de flujos turísticos dentro del espacio patrimonial; acciones para optimizar las relaciones con la población local; potenciación de la empleabilidad entre la población local con el recurso; diseño y ejecución de una política de conservación preventiva; apuesta por acciones que generen un mayor impacto económico. 


\section{Figura 3}

Valoración otorgada por académicos y profesionales

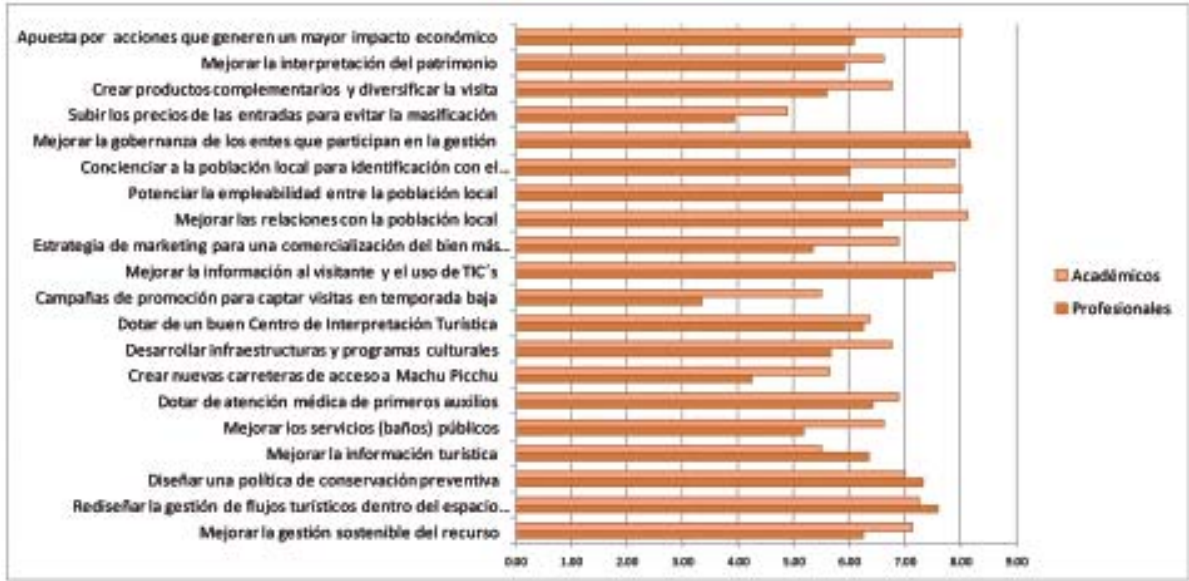

Nota. Elaboración propia.

- Acciones que tienen una valoración similar por los académicos y profesionales del sector turístico: mejora de la gobernanza de los entes que participan en la gestión; dotar de un buen centro de interpretación turística; rediseñar la gestión de flujos turísticos dentro del espacio patrimonial; necesidad de diseñar una política de conservación preventiva.

- Una valoración con mayor diferencia es la referida a la realización de campañas de promoción para captar visitas en temporada baja, con más de dos puntos entre la valoración otorgada por académicos (5.50) y la de profesionales (3.33).

- Otros aspectos con valoración diferente entre los dos grupos de expertos son los relacionados con los impactos sobre la población local. Con una alta valoración por los académicos y baja por los profesionales: acciones que generen un mayor impacto económico en el entorno tiene una puntuación de 8.00 por los académicos y de 6.08 por los profesionales; concienciación de la población local para identificación con el destino turístico tiene una puntuación de 7.88 por los académicos y de 6.00 por los profesionales.

En cuanto al grado de consenso, con respecto a las respuestas de los académicos y profesionales, se observa una coincidencia significativa; sin embargo, también se registran diferencias. A continuación se mencionan algunas:

- Con mayor divergencia, y que se reproducen con la misma tendencia, en los dos grupos de expertos (académicos y profesionales), se encuentran la puesta en marcha de accesos por carretera a Machu Picchu y la subida de precios de las entradas para evitar la masificación. 
- Se observa mayor convergencia en las opiniones de los profesionales que en las de los académicos, ya que en el global de las opiniones de profesionales su desviación estándar es de 1.14, mientras que las de los académicos es de 1.23 .

- Entre los académicos existen propuestas que conllevan a unas reflexiones más diversas, ya que tienen desviaciones estándar mayores a 1.5, además de las dos señaladas anteriormente, cuatro más: mejora de los servicios (baños) públicos; dotación de un buen centro de interpretación turística; realización de campañas de promoción para captar visitas en temporada baja; mejora de la infraestructura de interpretación del patrimonio.

Las medidas con mayor coincidencia, en las repuestas de los dos grupos de expertos participantes en la investigación, son cinco: crear productos complementarios y diversificar la visita; mejorar la información al visitante y el uso de las TIC; dotar de atención médica de primeros auxilios; diseñar una política de conservación preventiva y desarrollar infraestructuras y programas culturales.

\section{Figura 4}

Grado de consenso de académicos y profesionales

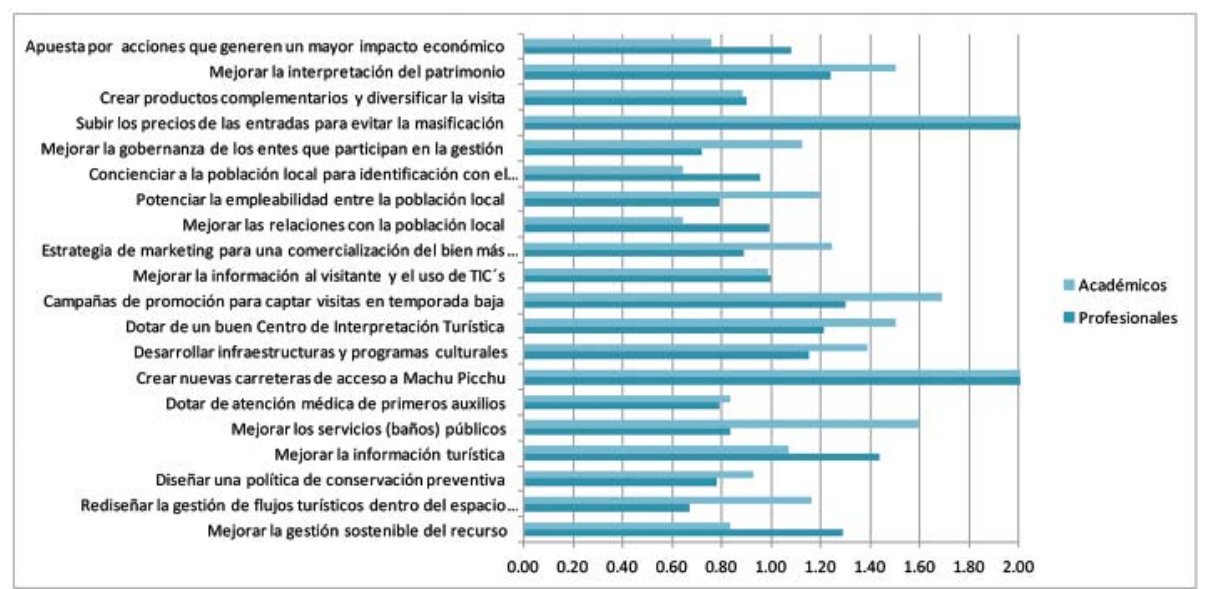

Nota. Elaboración propia. 


\section{Análisis según estrategias analizadas}

Las 20 propuestas de esta investigación se agrupan en seis estrategias con acción homogénea: gestión ambiental, gestión de los servicios turísticos, infraestructura, marketing, intervención pública y producto turístico. El conjunto de expertos entrevistados valoró los aspectos incluidos en cada bloque.

En la estrategia de gestión ambiental, los expertos entrevistados la señalan como la de mayor importancia entre los lineamientos para la mejora del SHM, con una valoración media de 7.08 sobre los 6.34 puntos de valoración media de las 20 propuestas planteadas. Además, se observa consenso en la opinión de los expertos entrevistados, ya que la desviación estándar para este tema es la menor en las seis estrategias planteadas. Por orden de valoración son las siguientes: rediseñar la gestión de flujos turísticos dentro del espacio patrimonial; planificación de una política de conservación preventiva; mejora de la gestión sostenible del recurso.

La gestión de los servicios turísticos recibe, de los expertos entrevistados, una puntuación intermedia de 6.12. También se observa un relativo consenso, ya que la desviación estándar obtenida de los servicios turísticos es de un aceptable 1.18. Por orden de valoración, las acciones de estrategias de servicios turísticos son las siguientes: creación de servicios de atención médica de primeros auxilios; mejora de la información turística; dotación en el interior del recurso de servicios (baños) públicos.

La estrategia sobre infraestructura es valorada, por el conjunto de expertos entrevistados, como de menor importancia entre los aspectos señalados para la mejora del SHM; con una valoración media de 5.73 sobre los 6.34 puntos de valoración media de las 20 propuestas planteadas. Las acciones relacionadas con infraestructura tienen mayor divergencia en las opiniones de los expertos. Por orden de valoración son las siguientes: dotación de un buen centro de interpretación turística; desarrollo de infraestructuras y programas culturales; planificación de nuevo acceso por carretera a Aguas Calientes, esta última, con la menor valoración y convergencia de los expertos.

Con respecto al marketing turístico, los expertos entrevistados, lo consideran de baja importancia entre los aspectos señalados para la mejora del SHM, con una valoración media de 5.93 sobre los 6.34 puntos de valoración media de las 20 propuestas planteadas. Es decir, la promoción del recurso no se plantea como prioritaria, ya que se dispone de demanda, al margen de las estrategias de captación. También se observa un consenso medio, ya que la desviación estándar obtenida de los aspectos ambientales es muy parecida a la obtenida en el trabajo en su conjunto. Las acciones de estrategia de marketing, por orden de valoración, son las siguientes: mejora de la información al visitante y el uso de las TIC; estrategia de marketing para una comercialización del bien más sostenible; realización de campañas de promoción para captar visitas en temporada baja.

En cuanto a intervención pública, el conjunto de expertos entrevistados lo considera como el de mayor importancia entre los aspectos señalados para la mejora del SHM con una valoración media de 7.31 sobre los 6.34 puntos de valoración media. Además, el grado de consenso es la segunda más baja (1.12 de desviación estándar) de las estrategias señaladas. Las acciones de la estrategia de intervención pública, por orden de valoración son las siguientes: coordinación eficiente de la gobernanza de los entes que participan en la gestión; mejora de las relaciones con la población local; potenciar la empleabilidad entre la población local; realizar acciones para concienciar a la población local para identificación con el destino turístico. 
Finalmente, en gestión de los productos turísticos, el conjunto de los expertos entrevistados lo valoran con una puntuación intermedia de 5.85. En el grado de consenso, la desviación estándar es la segunda mayor de los seis bloques de estrategias planteadas. Las acciones de estrategia sobre el producto turístico, por orden de valoración son las siguientes: acciones que generen un mayor impacto económico; mejorar la interpretación del patrimonio; crear productos complementarios y diversificar la visita; subir los precios de las entradas para evitar la masificación.

\section{Conclusiones}

El principal recurso turístico del Perú es, sin duda, el Santuario Histórico de Machu Picchu, por su capacidad para atraer turistas extranjeros, situación que podría replicarse con otros destinos del país.

Producto de la aplicación del método Delphy y del análisis en esta investigación empírica, se establece la importancia de implementar acciones para mejorar la competitividad del recurso SHM, así como el grado de consenso de dos grupos de expertos: los académicos y profesionales en la gestión turística.

Las principales medidas a implementar propuestas por los expertos entrevistados son las que tienen relación con la mejora de la gestión de los entes públicos a cargo del santuario; la mejora de la información que se pone a disposición del visitante a través de las TIC; la necesidad de rediseñar la gestión de los flujos turísticos dentro del espacio patrimonial; la necesidad de mejorar la política de conservación ambiental preventiva; y por último, la mejora de los efectos económicos y laborales, producto de las visitas y en favor de la población local.

Con menor valoración se encuentran la estrategia de marketing para una comercialización del bien más sostenible; la necesidad de mejorar los baños públicos; la creación de nuevas carreteras de acceso a Machu Picchu; la subida de los precios de las entradas para evitar la masificación; y la realización de campañas de promoción para captar visitas en temporada baja.

En cuanto al grado de coincidencia entre académicos y profesionales del sector turístico, en estos últimos, se detecta una mayor convergencia en las opiniones. La desviación estándar para los académicos es de 1.23; mientras que para los profesionales es de 1.14. En la puesta en marcha de accesos por carretera a Machu Picchu y la subida de precios de las entradas para evitar la masificación se evidencia una mayor diferencia.

Las acciones propuestas en las que se muestra una mayor coincidencia en las repuestas son las siguientes: creación de productos complementarios y diversificar la visita; mejora de la información al visitante mediante las tecnologías de la comunicación; la dotación de un servicio de atención médica de primeros auxilios; diseño de una política de conservación preventiva; desarrollar un programa de actividades culturales.

Por último, el conjunto de los expertos entrevistados, académicos y profesionales, valoran los aspectos ambientales como de mayor importancia entre los lineamientos señalados para la mejora del SHM, con una valoración media de 7.08 sobre 6.34 puntos de valoración media. Existe amplio consenso mostrado en que su desviación estándar es la menor de las seis tipologías de estrategias planteadas en la investigación. 


\section{Limitaciones y futuras investigaciones*}

Se consideran limitaciones a nivel metodológico y a nivel de gestión del SHM; en el primer caso por el uso del método Delphi, ya que según criterios estadísticos no otorga representatividad (Landeta et al., 2011). En el segundo caso, a nivel de gestión, es necesario señalar que los entrevistados conocen el recurso y lo han visitado en varias ocasiones, sin embargo, no lo analizan como expertos, sino como especialistas en el sector turístico que observan desde otro territorio la situación del santuario histórico.

Es frecuente aplicar técnicas cuantitativas en las investigaciones sobre gestión de recursos turísticos patrimoniales, dejando de lado técnicas cualitativas que también son de gran utilidad. Es necesario tener en cuenta las opiniones de académicos y profesionales en recursos turísticos con reconocido prestigio en su ámbito de trabajo, para que los procesos de puesta en valor de los recursos turísticos patrimoniales sean eficientes.

* Las diferentes fases de esta investigación (la revisión de literatura especializada, el trabajo de campo, la aplicación del método Delphi, así como la sistematización, análisis y procesamiento de la información) se llevaron a cabo durante 2019 y fueron concluidas en los primeros meses de 2020, antes del inicio de la pandemia ocasionada por la COVID-19; por esta razón no se incluyen temas relacionados. 


\section{Referencias}

Alva, W. (1996). El patrimonio arqueológico singular. Criterios para su explotación turística. El Impacto el Turismo en el Patrimonio Cultural [Seminario]. La Antigua, Guatemala.

Calderón, B. (2002). La Gestión del Patrimonio Cultural. La transmisión de un legado. Boletín de Arte, 23, 671-673. https://doi.org/ 10.24310/bolarte.2002.v0i23.4779

Cátedra Unesco Patrimonio Cultural y Turismo Sostenible. Universidad de San Martín de Porres. (2020). Santuario Histórico de Machu Picchu Patrimonio Mixto Natural y Cultural. http://catedraunesco.usmp.edu.pe/2018/08/21/santuario-historico-de-machu-picchupatrimonio-mixto-natural-y-cultural/

Consejo Nacional del Ambiente (CONAM). (2005). Reglamento de la Ley Marco del Sistema Nacional de Gestión Ambiental. Título primero. Disposiciones Generales, artículo 7 (p. 37). http://www2.produce.gob.pe/dispositivos/publicaciones/2005/ds008-2005-pcm.pdf

Cuadrao, C. y Advíncula, M. (2017). Problemáticas y alternativas en la gestión de un tramo del Camino Inca: el caso del tramo Vilcashuamán - Pisco, Qhapaq Ñan, Perú. Estudios Sobre Conservación, Restauración y Museología, 4, 159-170.

Dirección Desconcentrada de Cultura Cusco. (2020). Área Funcional del Parque Arqueológico Nacional de Machupicchu. Machu Picchu. https://www.culturacusco.gob.pe/area-funcional-del-parque-arqueologico-nacional-de-machupicchu/

Espinoza, O. y Gildemeister, M. (2017). Tejiendo nuevos modos de gestión del patrimonio cultural: el caso del proceso del saneamiento físico-legal de la ciudad inca de Vilcashuamán, Perú. Estudios Sobre Conservación, Restauración y Museología, 4, $121-134$.

Gómez, M. C. S. (2015). La dicotomía cualitativo-cuantitativo: posibilidades de integración y diseños mixtos. Campo Abierto. Revista de Educación, 11-30.

Hayakawa, J. (2009). Gestión del Patrimonio Cultural y Centros Históricos: Apuntes en Clave Latinoamericana. Turismo y Patrimonio, 6, 9-16.

Hernández, B. (2002). La gestión del patrimonio cultural. La transmisión de un legado. En M. Arias (coord.), La gestión del patrimonio cultural. La transmisión de un legado (pp. 13-15). [Introducción, ponencia inaugural]. Caja España, Fundación del Patrimonio Histórico de Castilla y León.

Katahenggam, N. (2020). Percepciones turísticas y preferencias de autenticidad en el turismo patrimonial. Revista de Turismo y Cambio Cultural, 18(4), 371-385.

Kauffman, F. (1988). Historia General de los Peruanos (t. 1). En P. E. Inca (Ed.). PEISA.

Landeta, J., Barrutia, J. y Lertxundi, A. (2011). Hybrid Delphi: A methodology to facilitate contribution from experts in professional contexts. Technological Forecasting and Social Change, 78(9), 1629-1641.

Ley n. ${ }^{\circ}$ 28296. Ley General del Patrimonio Cultural de la Nación. (2004). Ley General del Patrimonio Cultural de la Nación.

Lima Gris. (3 de enero de 2019a). Ingreso a Machu Picchu colapsa por el nuevo reglamento. https://limagris.com/ingreso-a-machupicchu-colapsa-por-el-nuevo-reglamento/

Lima Gris. (14 de octubre de 2019b). Turistas venden artesanía dentro de Machu Picchu. https://limagris.com/turistas-venden-artesaniadentro-de-machu-picchu/

Lopez, F., Torres-Delgado, A., Font, X. y Serrano, D. (2018). Gestión sostenible de destinos turísticos: la implementación de un sistema de indicadores de turismo en los destinos de la provincia de Barcelona. Boletín de la asociación de geógrafos españoles, 77, 428461. https://doi.org/: http://dx.doi.org/10.21138/bage.2547

Lozano, M. (2002). El consorcio de la ciudad monumental histórico-artística y arqueológica de Mérida. En M. Arias (coord.), La gestión del patrimonio cultural. La transmisión de un legado (pp. 49-70). Caja España, Fundación del Patrimonio Histórico de Castilla y León.

Lumbreras, L. G. (2000). Las formas históricas del Perú. Lluvia Editores.

Marcone, G. (2019). Entonces, el patrimonio existe? qué hacemos con el? Diálogos En Patrimonio Cultural n. ${ }^{\circ}$ 2, El Señuelo Patrimonial, 77-86. 
Martínez Carazo, P. C. (2011). El método de estudio de caso Estrategia metodológica de la investigación científica. Revista científica Pensamiento y Gestión, 20.

Martinez del Vas, G. (2017). Percepción del proceso de planificación estratégica como instrumento de la política turística a escala regional. Boletín de la asociación de geógrafos españoles, 75, 471-496. https://doi.org/10.21138/bage.2509

Ministerio de Economía y Finanzas (MEF). (s.f.). Sistema Nacional de Programación Multianual y Gestión de Inversiones INVIERTE.PE. https://www.mef.gob.pe/es/acerca-del-invierte-pe

Ministerio de Economía y Finanzas (MEF). (2011). Turismo. Guía para la formulación de proyectos de inversión exitosos. Guía Metodológica para la identificación, formulación y evaluación social de proyectos de inversión pública del sector turismo, a nivel de perfil.

Ministerio de Comerico Exterior y Turismo (MINCETUR). (2009). Ley General de Turismo n. 29408 (p. 8).

Ministerio de Comerico Exterior y Turismo (MINCETUR). (2020). Reporte Mensual del Turismo, diciembre 2019.

Ministerio de Cultura. (2011). Manual del Defensor del Patrimonio Cultural (3. ${ }^{\text {a ed.). }}$

Organización Mundial de Turismo (OMT). (2004). Desarrollo Sostenible del Turismo. Definición conceptual. https://www.unwto.org/ sustainable-developmentes/content/definicion

Organización Mundial de Turismo (OMT). (2016). Alianza entre turismo y cultura en el Perú, Modelos de colaboración entre turismo, cultura y comunidad. https://www.e-unwto.org/doi/pdf/10.18111/9789284417575

Pino, J. (2019). Entrevista a Jose Luis Pino Matos. Encargado de la Unidad de Conservación del Programa Qhapaq Ñan-Sede Nacional Del Ministerio De Cultura (Cátedra UNESCO). http://catedraunesco.usmp.edu.pe/2019/07/22/entrevista-jose-luis-pino/

Pulido, J. y Ruiz, A. (2017). Propuesta para una agenda de investigación de los impactos del turismo en los destinos Patrimonio de la Humanidad. Boletín de la asociación de geógrafos españoles, 73, 389-411. https://doi.org/10.21138/bage.2423

Servicio Nacional de Áreas Naturales Protegidas (SERNANP). (n.d.). De Machu Picchu. El Santuario Histórico de Machu Picchu. https://www.sernanp.gob.pe/de-machupicchu

World Economic Forum. (2013). The Peru Travel and Tourism Competitiveness. [Report 2013]. Ginebra.

Ypeij, A. (2020). Pellegrino A. Luciano, Neoliberal Reform in Machu Picchu: Protecting a Community, Heritage Site, and Tourism Destination in Peru (Lanham, MD y Londres: Lexington Books, 2018). Revista de Estudios Latinoamericanos, 52(2), 442-444.

\section{Rafael Fuentes García}

Doctor en Ciencias Económicas y Empresariales. Profesor de la Universidad de Málaga y Universidad Oberta o de la OMT-Academics, entre otras. Jefe del Área de Inteligencia Turística de Turismo y Planificación Costa del Sol. Especializado en el análisis del Sector Turístico, ámbito en los que desarrolla su labor profesional, investigadora y docente. Premio de Investigación Turística de Andalucía, de la Ciudad de Melilla, de Turismo de la Universidad de Málaga, embajador turístico de Mendoza (Argentina). Reconocimiento a la aportación al mundo académico Latinoamericano en materia turística. Escritor, director y coordinador de más de veinte publicaciones relacionadas con el sector turístico. Director de proyectos de planificación turística en 12 países.

fuentes@uma.es

ORCID: https://orcid.org/0000-0001-7251-4535

\section{Angélica Arriola Miranda}

Doctora en Turismo y Magister Scientiae en Ecoturismo. Profesora e investigadora de la Escuela de Turismo y Hotelería de la Universidad de San Martín de Porres. Ejerció el cargo de Técnico Nacional en el Proyecto «Desarrollo de capacidades locales para la implementación del Turismo Rural Comunitario integrando el Qhapaq Ñan en Bolivia, Perú y Ecuador» del Programa de las Naciones Unidas para el Desarrollo PNUD Perú, siendo responsable del diseño técnico y ejecución del proyecto a nivel nacional. Directora de Extensión Educativa del Cenfotur (2014). Directora Regional de Comercio Exterior y Turismo de Cajamarca (2011). Facilitadora para la Organización Mundial del Turismo en la dirección del Curso «Excelencia en la Gestión de los Recursos Humanos: Inclusión y Empleo Turístico» (Argentina, 2014). Becada por la Organización Mundial de Turismo para el curso «Gestión de Destinos Turísticos» (Oaxaca, México, 2013).

aarriolam@usmp.pe

ORCID: https://orcid.org/0000-0002-9495-327X 